\title{
A systematic review and meta-analysis of the safety and efficacy of anti-thymocyte globulin combined with eltrombopag in the treatment of severe aplastic anemia
}

\author{
Jiayou Zhang ${ }^{1}$, Yabing Wu ${ }^{1}$, Junhui Liu ${ }^{1}$, Shaolin Han ${ }^{1}$, Lijuan Chen ${ }^{1}$, Hui Wang ${ }^{2,3}$, Yu Peng ${ }^{3,4}$ \\ ${ }^{1}$ Hematology, 416 Hospital of Nuclear Industry, Second Affiliated Hospital of Chengdu Medical College, Chengdu, China; ${ }^{2}$ Department of Geriatric \\ Medicine One, Sichuan Provincial People's Hospital, University of Electronic Science and Technology of China, Chengdu, China; ${ }^{3}$ Chinese \\ Academy of Sciences Sichuan Translational Medicine Research Hospital, Chengdu, China; ${ }^{4}$ Department of Academic Journal, Sichuan Provincial \\ People's Hospital, University of Electronic Science and Technology of China, Chengdu, China \\ Contributions: (I) Conception and design: J Zhang, Y Wu, J Liu; (II) Administrative support: S Han; (III) Provision of study materials or patients: J \\ Zhang, Y Wu, J Liu, Y Peng, H Wang; (IV) Collection and assembly of data: All authors; (V) Data analysis and interpretation: J Zhang, L Chen, Y \\ Peng, H Wang; (VI) Manuscript writing: All authors; (VII) Final approval of manuscript: All authors. \\ Correspondence to: Yu Peng; Hui Wang. Sichuan Provincial People's Hospital, University of Electronic Science and Technology of China, Chengdu \\ 610072, China; Chinese Academy of Sciences Sichuan Translational Medicine Research Hospital, Chengdu 610072, China. \\ Email: pengyu5263@163.com; 407631627@qq.com.
}

Background: When it comes to the treatment of aplastic anemia fever, the Guidelines for Aplastic Anemia regards Anti-thymocyte globulin (ATG) combined with eltrombopag as the standard immunosuppressive treatment plan, and ATG is the main mode to treat severe aplastic anemia. A large number of prospective studies and clinical trials have confirmed the clinical application value of eltrombopag in aplastic anemia. Although ATG combined with eltrombopag brings satisfactory treatment results, the safety of long-term use is still unclear. Therefore, more clinical trial studies are needed to verify its safety.

Methods: Literature in the Chinese and English medical databases was searched using the following search terms: "Antithymocyte globulin", "severed aplastic anemia" and "eltrombopag". Patients in the experimental group were administered ATG combined with eltrombopag and patients in the control group received ATG treatment alone. Rev Man5.3 software was used for meta-analysis.

Results: A total of 16 references were included in this meta-analysis. Heterogeneity tests examining total effective rate demonstrated that $\mathrm{Chi}^{2}=4.48, \mathrm{df}=15, \mathrm{I}^{2}=0 \%<50 \%$, and $\mathrm{P}=1.00>0.01$. The effective rate of the experimental group was higher than that of the control group, with odds ratio $(\mathrm{OR})=1.90$ and $95 \%$ confidence interval (CI) 1.35 to $2.68(\mathrm{Z}=3.70, \mathrm{P}=0.0002)$. The heterogeneity test results of the survival rate within 2 years were $\mathrm{Chi}^{2}=3.09, \mathrm{df}=7, \mathrm{I}^{2}=0 \%<50 \%$, and $\mathrm{P}=0.88>0.01$. The survival rate of the experimental group was higher than that of the control group, with $\mathrm{OR}=2.54$, and $95 \% \mathrm{CI}: 1.58$ to $4.09(\mathrm{Z}=3.84, \mathrm{P}=0.0001)$. The heterogeneity test results of the mortality rate were $\mathrm{Chi}^{2}=3.49, \mathrm{df}=6, \mathrm{I}^{2}=0 \%<50 \%$, and $\mathrm{P}=0.75>0.01$. The mortality rate of the experimental group was lower than that of the control group, with $\mathrm{OR}=0.48$ and 95\% CI: 0.33 to $0.70(\mathrm{Z}=3.84, \mathrm{P}=0.0001)$. The heterogeneity test results of the occurrence of side effects were $\mathrm{Chi}^{2}=0.12$, df $=3, \mathrm{I}^{2}=0 \%<50 \%, \mathrm{P}=0.99>0.01$. The incidence of side effects in the experimental group was lower than that in the control group, with $\mathrm{OR}=0.74,95 \% \mathrm{CI}: 0.48$ to $1.17(\mathrm{Z}=1.29, \mathrm{P}=0.20)$.

Discussion: This meta-analysis demonstrated that the combination of ATG with eltrombopag in the treatment of SAA is safer and more effective than ATG alone.

Keywords: Anti-thymocyte globulin (ATG); eltrombopag; severe aplastic anemia (SAA); safety

Submitted Mar 18, 2021. Accepted for publication May 19, 2021.

doi: 10.21037/apm-21-1049

View this article at: http://dx.doi.org/10.21037/apm-21-1049 


\section{Introduction}

Aplastic anemia (AA) refers to bone marrow failure syndrome due to the combined action of chemical, biological, and other factors. The main clinical manifestations are anemia, repeated infections, decreased bone marrow nuclear cell division, and reduced pancytopenia count (1). The incidence of AA can be as high as $75 \%$ in China (2). According to the disease condition, AA can be divided into severe aplastic anemia (SAA) and mild aplastic anemia (MAA) (3). SAA has an acute onset with a mortality rate of $90 \%$. Patients with SAA usually require regular blood transfusions which often lead to severe bleeding or infections. Since SAA is largely due to damage of the bone marrow stem cells and the hematopoietic internal environment, immunosuppressive agents have been used in the treatment of acute AA (4).

Anti-thymocyte globulin (ATG) therapy uses T lymphocytes in the human body as antigens to immunize animals such as rabbits, horses, and pigs. These immunized animals then produce the corresponding specific antibodies against the donor $\mathrm{T}$ lymphocytes (5). These specific immunoglobulins against $\mathrm{T}$ lymphocytes are harvested and used to remove abnormal $\mathrm{T}$ lymphocytes in patients with AA, via cell apoptosis. Thus, normal hematopoietic function is restored (6).

In the 1970s, Yang et al. (7) proposed that ATG exerts remarkable therapeutic effects in patients with SAA and in recent years, ATG has become the main mode of treatment for SAA. In fact, studies have reported that ATG has an effective rate of $55 \%$ in treating SAA. The main side effects reported were bleeding and serum sickness. Eltrombopag is a drug that can enhance the regulation of $\mathrm{T}$ cells and $B$ cells, increase the secretion of transforming growth factor (TGF)- $\beta$, and reduce the release of tumor necrosis factor (TNF)- $\alpha$ (8). Interestingly, the application of ATG combined with eltrombopag increased the effective rate of SAA to about $70 \%$. Lengline et al. (9) found that $15 \%$ of patients recovered completely, whereas those of a high recurrence rate accounted for $33 \%$.

Although the combined administration of ATG with eltrombopag has significantly improved the prognosis of patients, the mortality rate from associated bleeding and infections is still $30 \%$ (10). To further explore the safety and efficacy of these two drugs in the treatment of SAA, the Cochrane system was adopted to scientifically evaluate randomized controlled trials (RCTs) published in the literature. This will provide a theoretical basis for the clinical application of ATG and eltrombopag in the treatment of SAA.

We present the following article in accordance with the PRISMA reporting checklist (available at http://dx.doi. org/10.21037/apm-21-1049).

\section{Methods}

\section{Literature retrieve}

PubMed, Medline, Cochrane Library, Chinese Biomedical Literature Database, CNKI (China National Knowledge Infrastructure) database, WANFANG database, VIP database, and Google Scholar were searched from the establishment of the database to December 20, 2020. The Boolean logic retrieval method was used to retrieve relevant references. In the Chinese databases, a combination of the following search terms was used: "anti-thymocyte globulin", "severe aplastic anemia", and "eltrombopag". In the English databases, "anti-thymocyte globulin", "severe aplastic anemia", and "eltrombopag" were used as the search terms. The quality of the literature was evaluated according to the Rev Man 5.3 software provided by the Cochrane system.

Each database adopted a joint search strategy of free words and subject words. After confirmation, the references were tracked using the search engine, and the latest research progress was obtained by contacting experts and researchers in the field.

\section{Literature inclusion and exclusion criteria}

The literature was selected according to the following inclusion criteria: (I) studies related to the treatment of SAA using ATG combined with eltrombopag; (II) RCTs; (III) pathological control analysis showed a reliability index of $95 \%$ confidence interval (CI); (IV) articles were in the Chinese or English language; and (V) the diagnosis of SAA met the standards of the World Health Organization.

The following studies were excluded: (I) literature not relevant to this study; (II) duplicate publications; (III) review articles, literature abstracts, case reports, and animal experiments; (IV) literature with incomplete data; and (V) literature in languages other than Chinese or English.

Two senior experts independently screened the abstracts and full text articles. In the case of disagreements, a consensus conclusion was obtained through discussion, or a third expert was invited to arbitrate. 


\section{Evaluation index}

The evaluation indexes included total effective rate (complete reaction and partial reaction were both defined as effective reaction), final mortality rate, survival rate within 2 years, side effects, and recurrence rate.

\section{Data extraction}

Two experts independently collated the data using a standardized Excel table. Three preliminary experiments were performed before extraction. Any inconsistencies were resolved through discussion or arbitration by a third expert. The following data were collated: (I) the title of the research; (II) the first author; (III) the name of the publication; (IV) year of publication; (V) the basic characteristics of the patient including age, gender, treatment plan, and drug dosage; (VI) the grouping methods and statistical methods applied; and (VII) the source, sample size, and outcome indexes of the cases.

\section{Bias risk assessment and quality evaluation}

Two researchers conducted a risk assessment of bias simultaneously. Any inconsistencies were resolved via discussion or arbitration by a third expert. In this study, the Cochrane Collaboration for "bias risk assessment" was used for RCTs. The assessments of "low risk bias", "unclear", and "high risk bias" were made according to the five aspects of random allocation method, blind method, allocation concealment, complete data, and research results.

The Newcastle-Ottawa Scale (NOS) was used to evaluate the quality of the literature according to three aspects, namely, patient selection, comparability of the study, and outcome. The score ranged from 0 to 9 . References with a score of 1 or less were considered low quality (high risk bias); references with a score of 2-5 were considered medium quality (unclear); and references with a score of 6-9 were considered high-quality literature (low risk bias).

\section{Statistical analysis}

StataSE12.0 software was used for statistical analyses. Odd ratios (ORs) and $95 \%$ confidence intervals $(\mathrm{CI})$ were used to evaluate the blood response rate, mortality rate, survival rate, and side effects of ATG combined with eltrombopag in the treatment of SAA. The Rev Man5.3 software was used to assess the bias risk of the included references. The effect was expressed using a $95 \%$ CI. When $\mathrm{P}>0.1$ and $\mathrm{I}^{2}<50 \%$, the fixed effects model was used for meta-analysis. When $\mathrm{P}<0.1$ and $\mathrm{I}^{2}>50 \%$, the random effects model was used for meta-analysis.

\section{Results}

\section{Basic information of included references}

Initially, a total of 1,753 references were identified, and 1,717 were excluded after reading the abstract and the title. A further 20 studies were excluded after reading the full text article. References were excluded due to duplicate research data [985], data which did not include case-control analyses [324], patients who did not undergo anesthesia surgery for liver cancer [394], and failure to extract relevant information [18] (Figure 1). Finally, 16 studies were included in this meta-analysis.

Figure 2 shows the quality classification results. There were 8 references with a score of 6-9 and above, 5 articles with a score of $2-5$, and 3 studies with a score of 2 and below.

Of the 16 references that met the inclusion criteria, 12 were retrospective analyses and 4 were RCTs, with a total of 1,264 patients. All 16 studies had an ATG control group with sample sizes ranging from 28 to 54 . These studies were analyzed for the number of cases, intervention measures, and observation indexes. The general characteristics of the research subjects are shown in Table 1 .

\section{The risk bias assessment}

Figures 3 and 4 show the risk bias assessment using Review Manager 5.3 software. Of the 16 RCTs involved in this study, $3(11,12,13)$ had the correct random allocation method, and only $2(14,15)$ had allocation concealment. One (16) reference adopted the blinding method, which was not used in the other studies. However, the measurement indexes were laboratory indexes determined by computer and hence, it can be considered that all references adopted the blinding method.

\section{The total effective rate}

The total effective rate in the RCTs was analyzed. There were a total of 1,264 cases, with 638 patients in the experimental group, and 626 patients in the control group. The heterogeneity test results with the fixed effects 


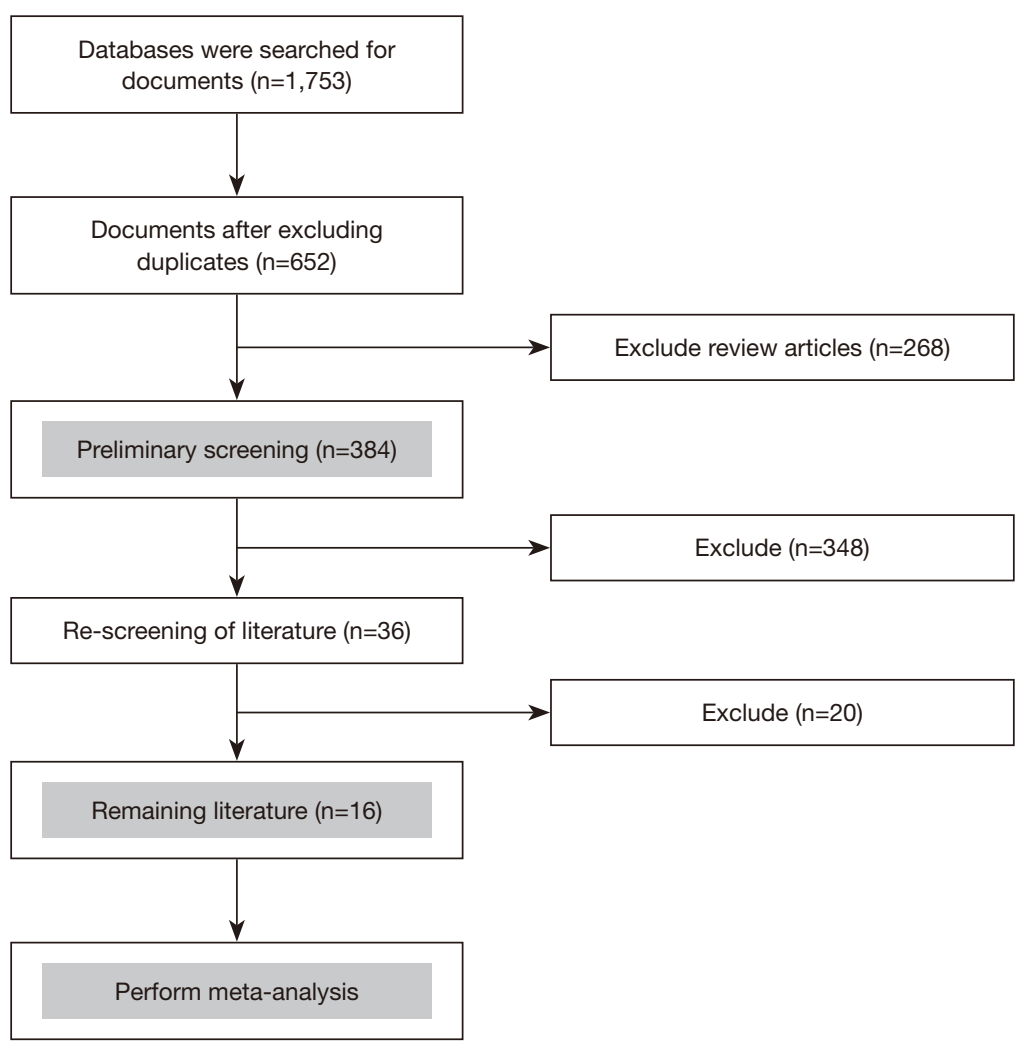

Figure 1 A flow chart depicting the literature search process.

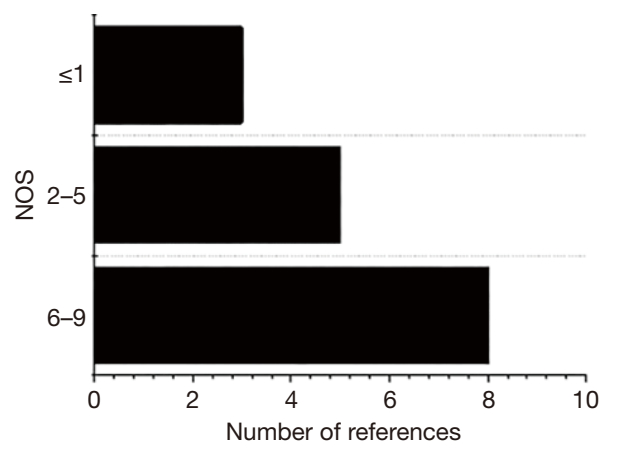

Figure 2 Literature quality classification results using the Newcastle-Ottawa Scale (NOS).

model revealed that $\mathrm{Chi}^{2}=4.48$, df $=15, \mathrm{I}^{2}=0 \%<50 \%$, and $\mathrm{P}=1.00>0.01$. The horizontal line and the invalid vertical line crossed at $95 \% \mathrm{CI}$ : in all the studies. Meta-analysis showed that the effective rate of ATG combined with eltrombopag was higher than that of ATG alone $(\mathrm{OR}=1.90$, $95 \% \mathrm{CI}: 1.35$ to 2.68$)$, and the difference was notable $(\mathrm{Z}=3.70, \mathrm{P}=0.0002$; Figure 5$)$.

The Rev Man 5.3 was used to obtain the funnel chart for the total effective rate (Figure 6). The circles in some studies were basically symmetrical to the midline, suggesting that the research accuracy was high and there was no publication bias.

\section{The survival rate}

There were 8 RCTs which analyzed the 2-year survival rate. There was a total of 642 cases, with 324 patients in the experimental group, and 318 patients in the control group. The heterogeneity test using the fixed effects model demonstrated that $\mathrm{Chi}^{2}=3.09$, $\mathrm{df}=7, \mathrm{I}^{2}=0 \%<50 \%$, and $\mathrm{P}=0.88>0.01$. The horizontal line and the invalid vertical line crossed at $95 \%$ CI: for all studies. Meta-analysis showed that the survival rate of patients in the ATG combined with eltrombopag group was higher than that of patients in the ATG alone group (OR $=2.54$ and $95 \% \mathrm{CI}$ : 1.58 to 4.09), and the difference was significant $(\mathrm{Z}=3.84, \mathrm{P}=0.0001$; Figure 7).

The Rev Man5.3 was used to obtain a funnel chart of the survival rate (Figure 8). The circles in some studies were basically symmetrical to the midline, suggesting that the research accuracy was high and there was no publication bias. 
Table 1 General characteristics of the research subjects

\begin{tabular}{|c|c|c|c|c|c|c|}
\hline Author & $\begin{array}{c}\text { Year of } \\
\text { publication }\end{array}$ & \multicolumn{2}{|c|}{ Number of cases } & \multicolumn{2}{|l|}{ Intervention } & Observation index \\
\hline Scheinberg $\mathrm{P}$ & 2011 & 48 & 48 & ATG + Eltrombopag & ATG & $\begin{array}{l}\text { Total effective rate ( } 3-6 \text { months), survival rate, } \\
\text { mortality, side effects }\end{array}$ \\
\hline Bacigalupo A & 2019 & 46 & 44 & ATG + Eltrombopag & ATG & $\begin{array}{l}\text { Total effective rate, survival rate, infection } \\
\text { (incidence rate, related death) }\end{array}$ \\
\hline Tichelli A & 2020 & 32 & 34 & ATG + Eltrombopag & ATG & $\begin{array}{l}\text { Total effective rate, adverse reaction over } 1 \text { year, } \\
\text { survival rate over } 2 \text { years }\end{array}$ \\
\hline Rogers ZR & 2019 & 26 & 26 & ATG + Eltrombopag & ATG & Total effective rate, mortality rate, basic cure rate \\
\hline Hayakawa J & 2017 & 52 & 50 & ATG + Eltrombopag & ATG & $\begin{array}{l}\text { Total effective rate, basic cure rate, recurrence } \\
\text { rate }\end{array}$ \\
\hline Sasaki N & 2019 & 42 & 40 & ATG + Eltrombopag & ATG & Total effectiveness, mortality rate, side effects \\
\hline Li F & 2020 & 48 & 48 & ATG + Eltrombopag & ATG & $\begin{array}{l}\text { Total effective rate, basic cure rate, recurrence } \\
\text { rate }\end{array}$ \\
\hline Sharma R & 2012 & 36 & 38 & ATG + Eltrombopag & ATG & $\begin{array}{l}\text { Total effective rate, mortality rate, recurrence } \\
\text { rate }\end{array}$ \\
\hline Bevans MF & 2004 & 40 & 40 & ATG + Eltrombopag & ATG & Total effective rate, average onset time \\
\hline Frickhofen $\mathrm{N}$ & 2000 & 26 & 26 & ATG + Eltrombopag & ATG & Total effective rate, mortality rate, basic cure rate \\
\hline Chandra J & 2008 & 54 & 52 & ATG + Eltrombopag & ATG & $\begin{array}{l}\text { Total effective rate, average onset time, side } \\
\text { effects }\end{array}$ \\
\hline Tang X & 2012 & 38 & 38 & ATG + Eltrombopag & ATG & Total effective rate, recurrence rate, side effects \\
\hline
\end{tabular}

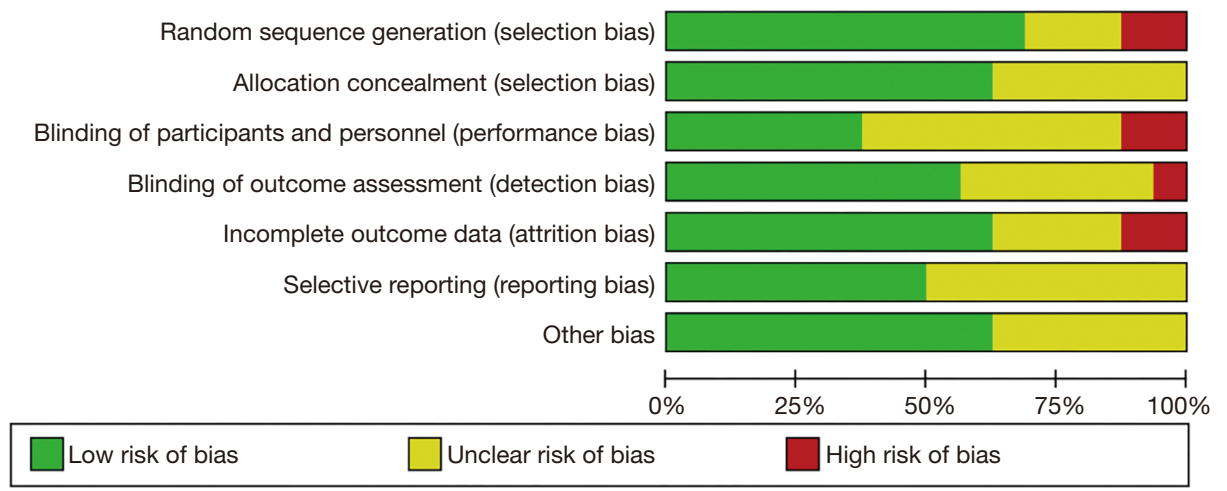

Figure 3 The bias risk evaluation results of the included literature. 


\section{The mortality rate}

There were 7 RCTs analyzing the final mortality rate, including a total of 514 cases, with 260 patients in the experimental group and 254 patients in the control group. The heterogeneity test using the fixed effects model showed that $\mathrm{Chi}^{2}=3.49, \mathrm{df}=6, \mathrm{I}^{2}=0 \%<50 \%$, and $\mathrm{P}=0.75>0.01$. In most studies, the horizontal line and the left side of the invalid vertical line crossed at $95 \% \mathrm{CI}$, and some were on the left side of the invalid vertical line. Meta-analysis demonstrated that the mortality rate of patients in the ATG combined with eltrombopag group was lower than that of patients in the ATG alone group (OR $=0.48$ and $95 \% \mathrm{CI}$ : 0.33 to 0.70$)$, and the difference was significant $(\mathrm{Z}=3.84$, $\mathrm{P}=0.0001$; Figure 9).

The Rev Man5.3 was used to obtain a mortality funnel chart (Figure 10). The circles in some studies were basically symmetrical to the midline, suggesting that the research accuracy was high and there was no publication bias.

\section{Side effects of treatment}

A total of four RCTs analyzed the side effects of treatment. There were a total of 360 cases, with 182 patients in the experimental group, and 178 patients in the control group. The heterogeneity test using the fixed effects model demonstrated that $\mathrm{Chi}^{2}=0.12, \mathrm{df}=3, \mathrm{I}^{2}=0 \%<50 \%$, and $\mathrm{P}=0.99>0.01$. The horizontal line and the left side of the invalid vertical line crossed at the $95 \%$ CI for all studies. Meta-analysis results showed that the incidence of side effects in patient treated with ATG combined with eltrombopag was lower than that in patients treated with ATG alone (OR $=0.74,95 \% \mathrm{CI}: 0.48$ to $1.17, \mathrm{Z}=1.29$, $\mathrm{P}=0.20$; Figure 11).

The Rev Man5.3 was used to obtain the funnel chart for side effects (Figure 12). The circles in some studies were basically symmetrical to the midline, suggesting that the research accuracy was high and there was no publication bias.

\section{The recurrence rate}

There were six RCTs analyzing the rate of SAA recurrence. There were 470 cases in total, with 236 patients in the experimental group and 234 patients in the control group. The heterogeneity test using the fixed effects model showed that $\mathrm{Chi}^{2}=1.01, \mathrm{df}=5, \mathrm{I}^{2}=0 \%<50 \%$, and $\mathrm{P}=0.96>0.01$. In most studies, the horizontal line and the invalid vertical line crossed at the $95 \%$ CI. The meta-analysis showed that compared with the ATG group, the recurrence rate

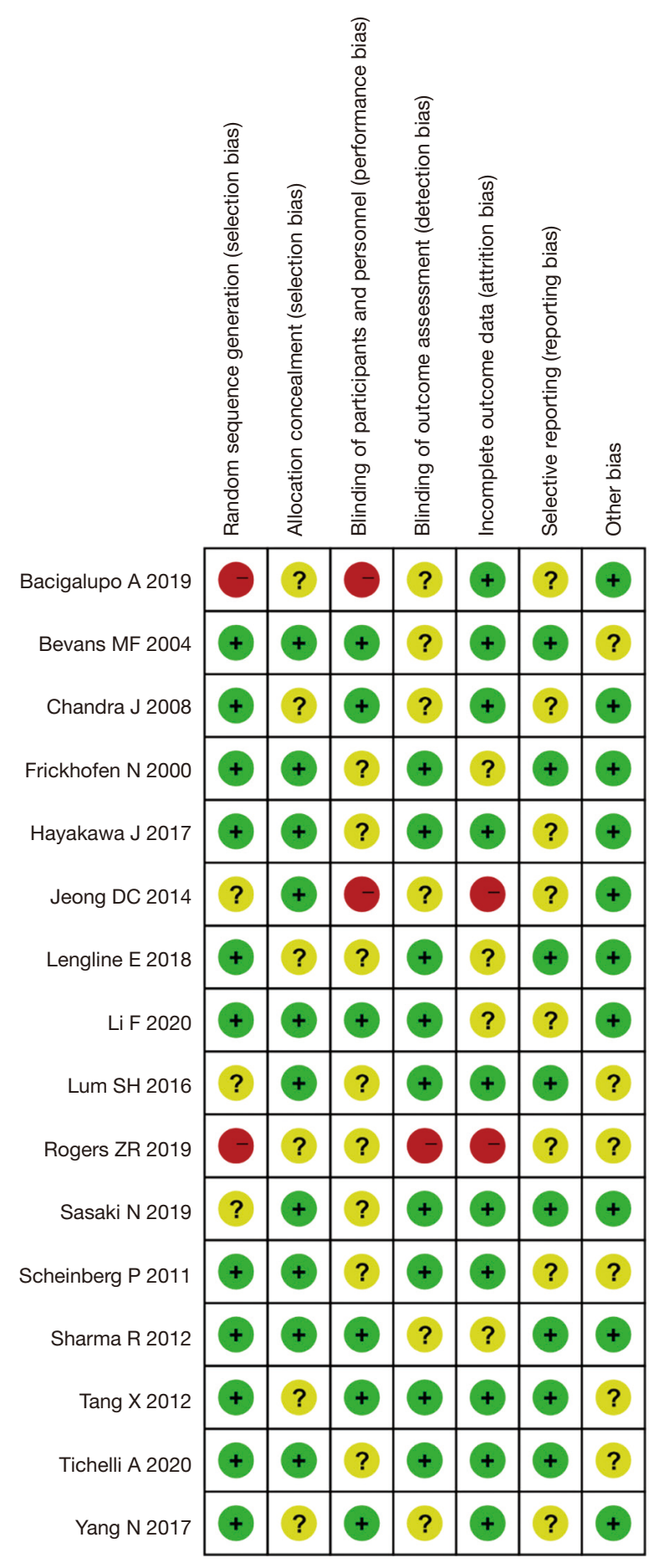

Figure 4 The multiple risk bias evaluation results of the included literature.

of patients treated with ATG combined with eltrombopag group was slightly lower (OR $=0.95$ and $95 \%$ CI: 0.66 to 1.37), however, the difference was not statistically significant 


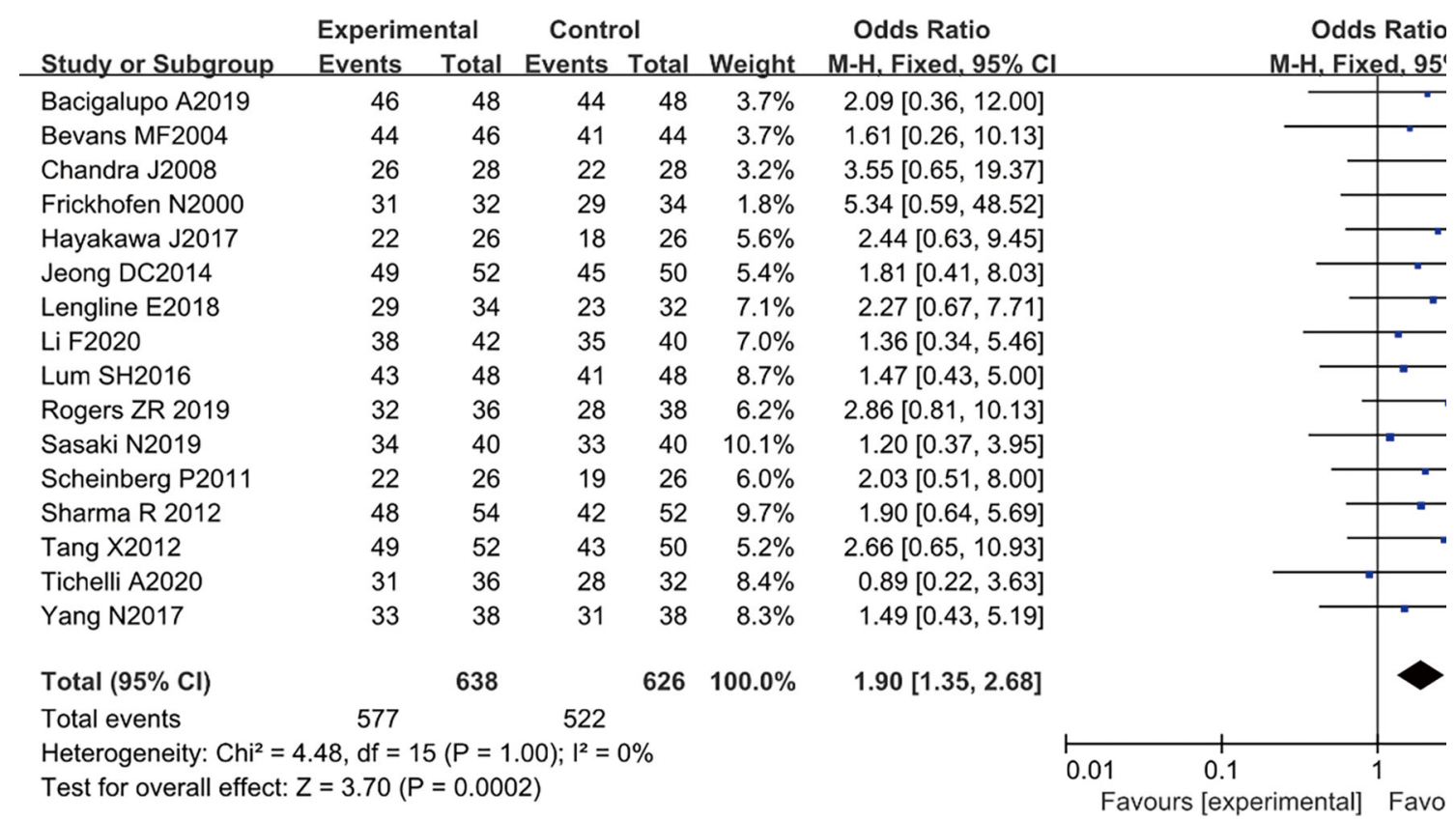

Figure 5 A forest plot showing the total effective rate between patients administered anti-thymocyte globulin (ATG) combined with eltrombopag and patients given ATG alone.

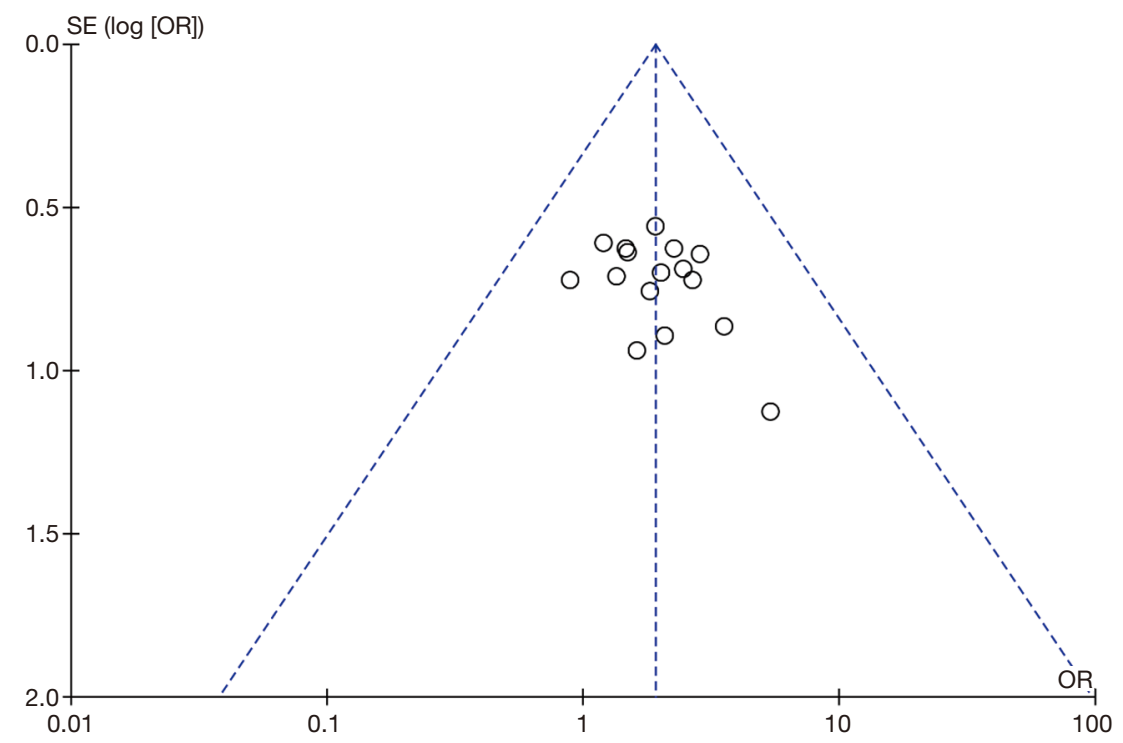

Figure 6 A funnel chart showing the total effective rate between patients administered anti-thymocyte globulin (ATG) combined with eltrombopag and patients given ATG alone.

( $\mathrm{Z}=0.29, \mathrm{P}=0.77$; Figure 13).

The Rev Man5.3 was used to obtain a funnel chart of the recurrence rate (Figure 14). It was evident that the circles in some studies were not symmetrical along the midline, suggesting that the research accuracy was low and the publication may be biased.

\section{Discussion}

ATG is a biologically active protein obtained by injecting 


\begin{tabular}{|c|c|c|c|c|c|}
\hline \multirow[b]{2}{*}{ Study or Subgroup } & \multicolumn{2}{|c|}{ Experimental } & \multicolumn{2}{|c|}{ Control } & \multirow[b]{2}{*}{ Weight } \\
\hline & Events & Total & Events & Total & \\
\hline Bacigalupo A2019 & 43 & 46 & 40 & 44 & $11.9 \%$ \\
\hline Frickhofen N2000 & 24 & 26 & 18 & 26 & $6.2 \%$ \\
\hline Hayakawa J2017 & 48 & 52 & 42 & 50 & $14.7 \%$ \\
\hline Lum SH2016 & 50 & 52 & 41 & 50 & $7.2 \%$ \\
\hline Rogers ZR 2019 & 18 & 26 & 15 & 26 & $20.6 \%$ \\
\hline Sasaki N2019 & 39 & 42 & 32 & 40 & $10.5 \%$ \\
\hline Scheinberg P2011 & 44 & 48 & 41 & 48 & $15.3 \%$ \\
\hline Tichelli A2020 & 28 & 32 & 25 & 34 & $13.6 \%$ \\
\hline Yang N2017 & 0 & 0 & 0 & 0 & \\
\hline Total $(95 \% \mathrm{Cl})$ & & 324 & & 318 & $100.0 \%$ \\
\hline Total events & 294 & & 254 & & \\
\hline \multirow{2}{*}{\multicolumn{6}{|c|}{ Heterogeneity: $\mathrm{Chi}^{2}=3.09, \mathrm{df}=7(\mathrm{P}=0.88) ; \mathrm{I}^{2}=0 \%$}} \\
\hline Test for overall effect & $=3.84(P$ & $=0.000$ & & & \\
\hline
\end{tabular}

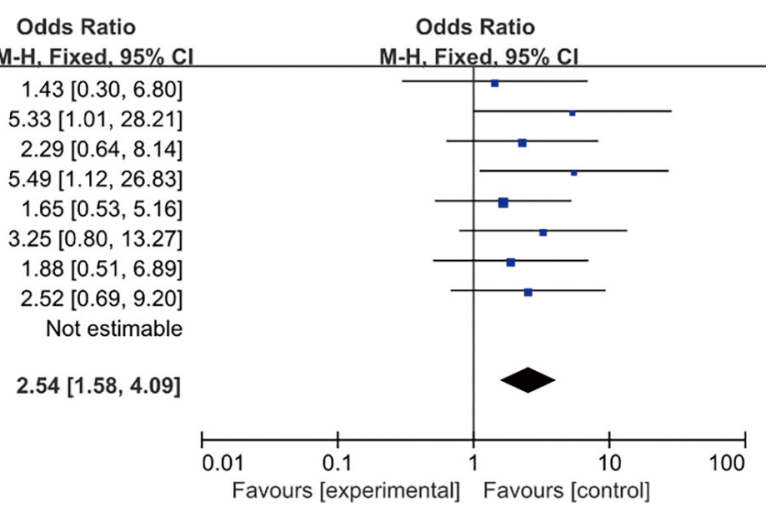

Figure 7 A comparison of the survival rates between patients administered anti-thymocyte globulin (ATG) combined with eltrombopag and patients given ATG alone.

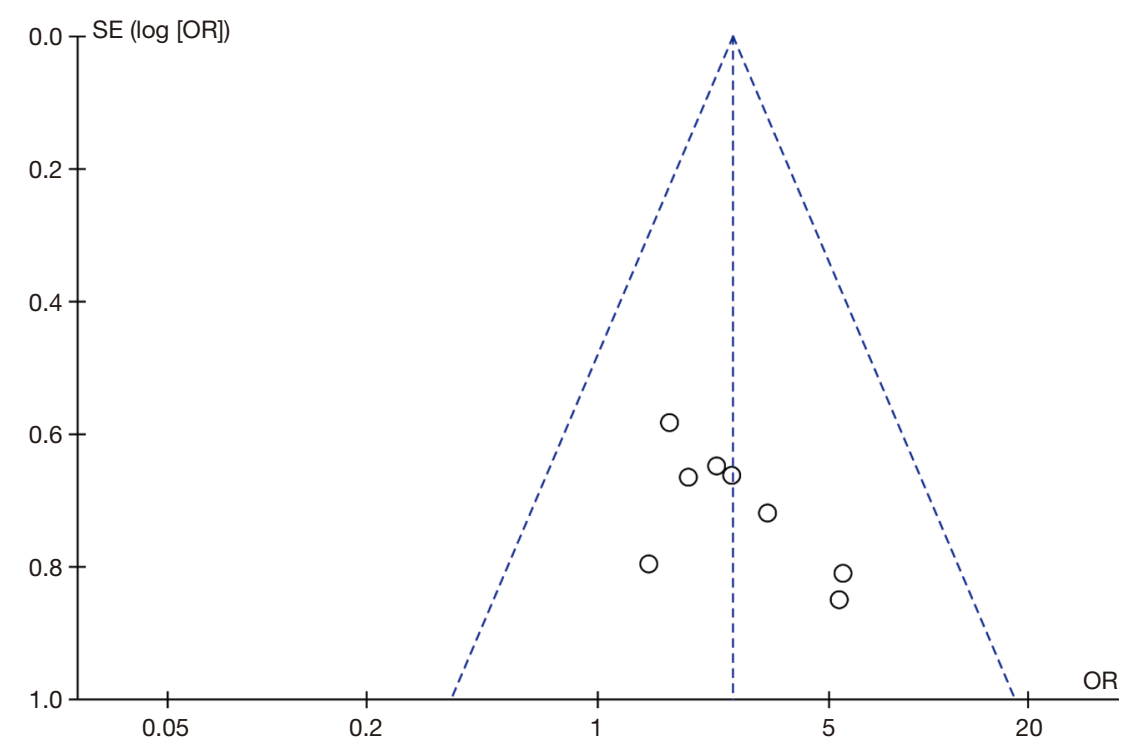

Figure 8 A funnel chart showing the survival rate between patients administered anti-thymocyte globulin (ATG) combined with eltrombopag and patients given ATG alone.

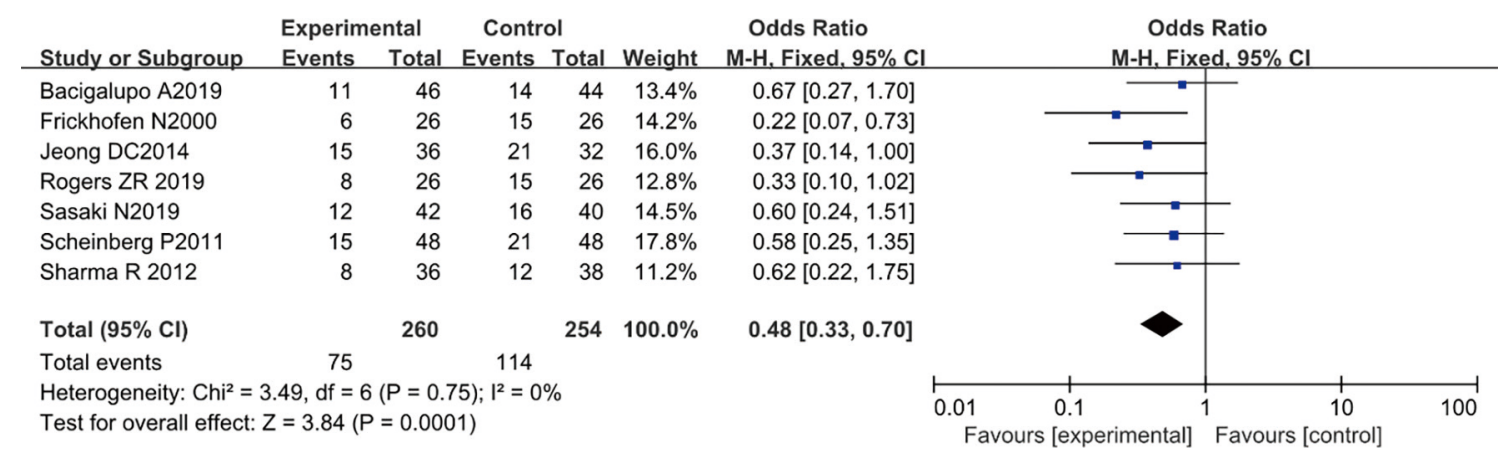

Figure 9 A forest plot showing the mortality rate between patients administered anti-thymocyte globulin (ATG) combined with eltrombopag and patients given ATG alone. 


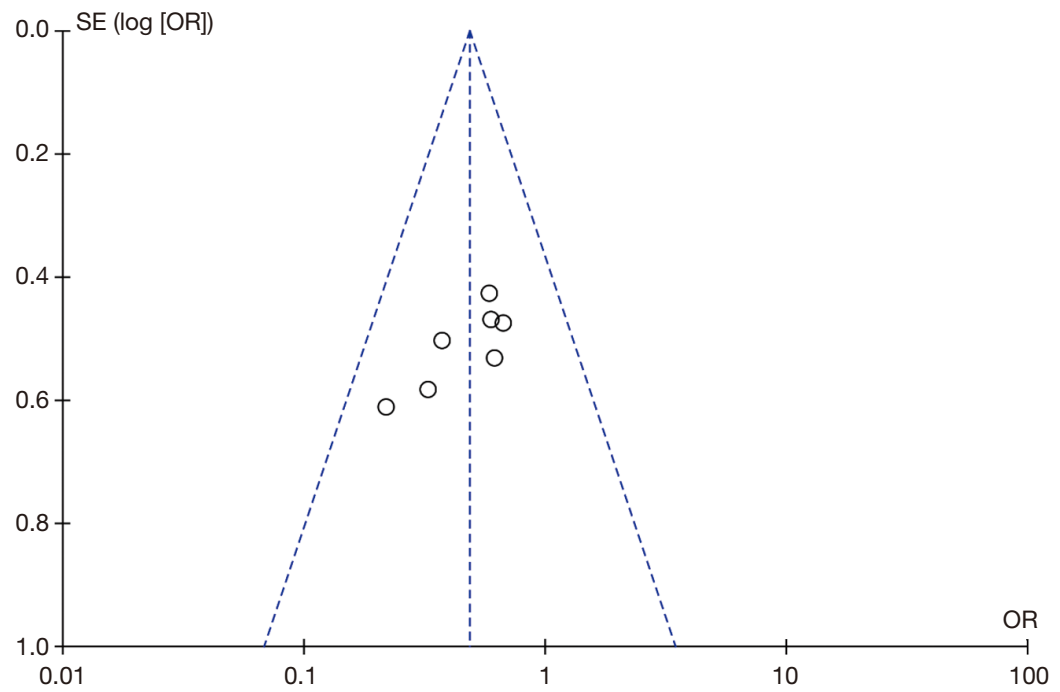

Figure 10 A funnel chart showing the mortality rate between patients administered anti-thymocyte globulin (ATG) combined with eltrombopag and patients given ATG alone.

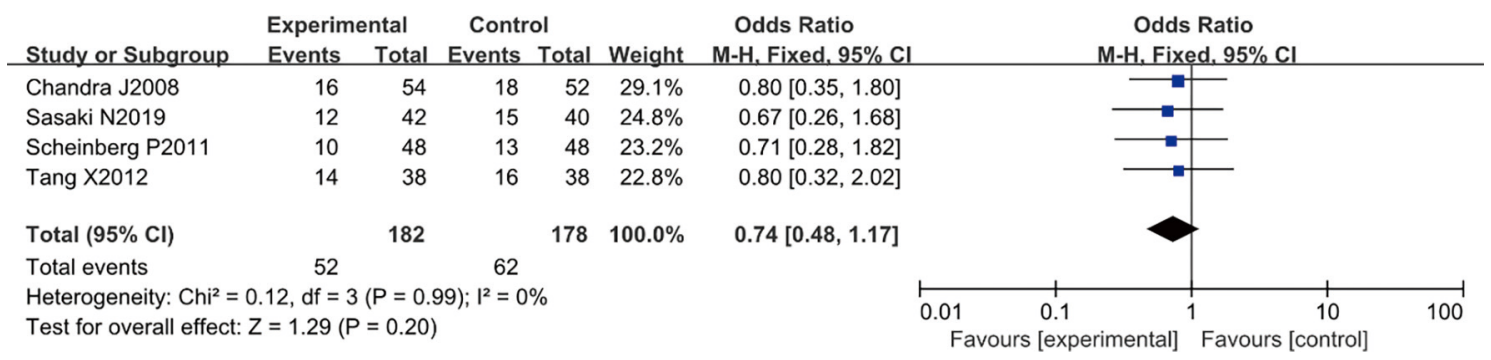

Figure 11 A forest plot showing the side effects between patients administered anti-thymocyte globulin (ATG) combined with eltrombopag and patients given ATG alone.

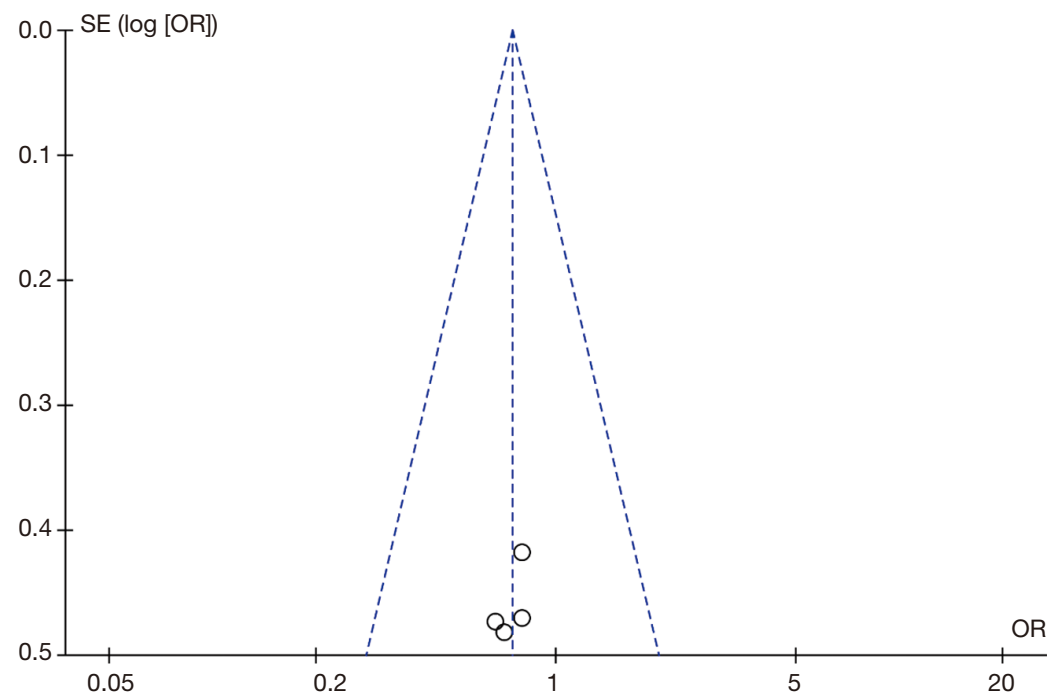

Figure 12 A funnel chart showing the side effects patients administered anti-thymocyte globulin (ATG) combined with eltrombopag and patients given ATG alone. 


\begin{tabular}{|c|c|c|c|c|c|}
\hline \multirow[b]{2}{*}{ Study or Subgroup } & \multicolumn{2}{|c|}{ Experimental } & \multicolumn{2}{|c|}{ Control } & \multirow[b]{2}{*}{ Weight } \\
\hline & Events & Total & Events & Total & \\
\hline Hayakawa J2017 & 28 & 52 & 27 & 50 & $21.4 \%$ \\
\hline Lengline E2018 & 15 & 34 & 16 & 32 & $15.5 \%$ \\
\hline Li F2020 & 26 & 48 & 27 & 48 & $20.9 \%$ \\
\hline Sharma R 2012 & 18 & 36 & 16 & 38 & $13.1 \%$ \\
\hline Tang X2012 & 14 & 38 & 15 & 38 & $16.0 \%$ \\
\hline Yang N2017 & 10 & 28 & 12 & 28 & $13.0 \%$ \\
\hline Total $(95 \% \mathrm{Cl})$ & & 236 & & 234 & $100.0 \%$ \\
\hline Total events & 111 & & 113 & & \\
\hline \multicolumn{6}{|c|}{ Heterogeneity: $\mathrm{Chi}^{2}=1.01, \mathrm{df}=5(\mathrm{P}=0.96) ; \mathrm{I}^{2}=0 \%$} \\
\hline Test for overall effect & $=0.29$ & $=0.77)$ & & & \\
\hline
\end{tabular}

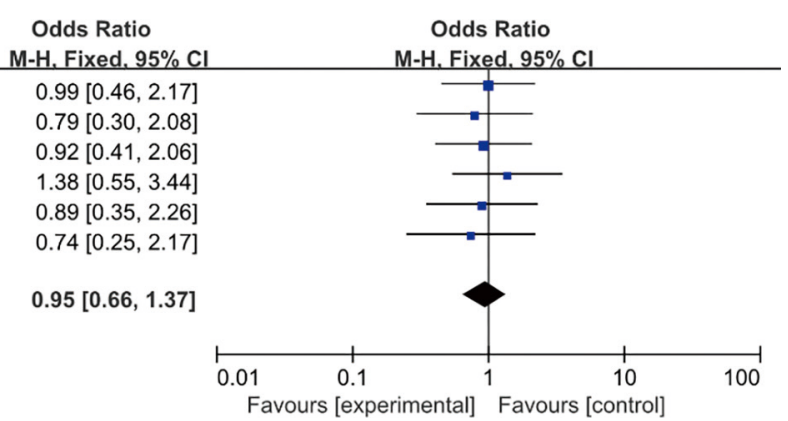

Figure 13 A forest plot showing the recurrence rate between patients administered anti-thymocyte globulin (ATG) combined with eltrombopag and patients given ATG alone.

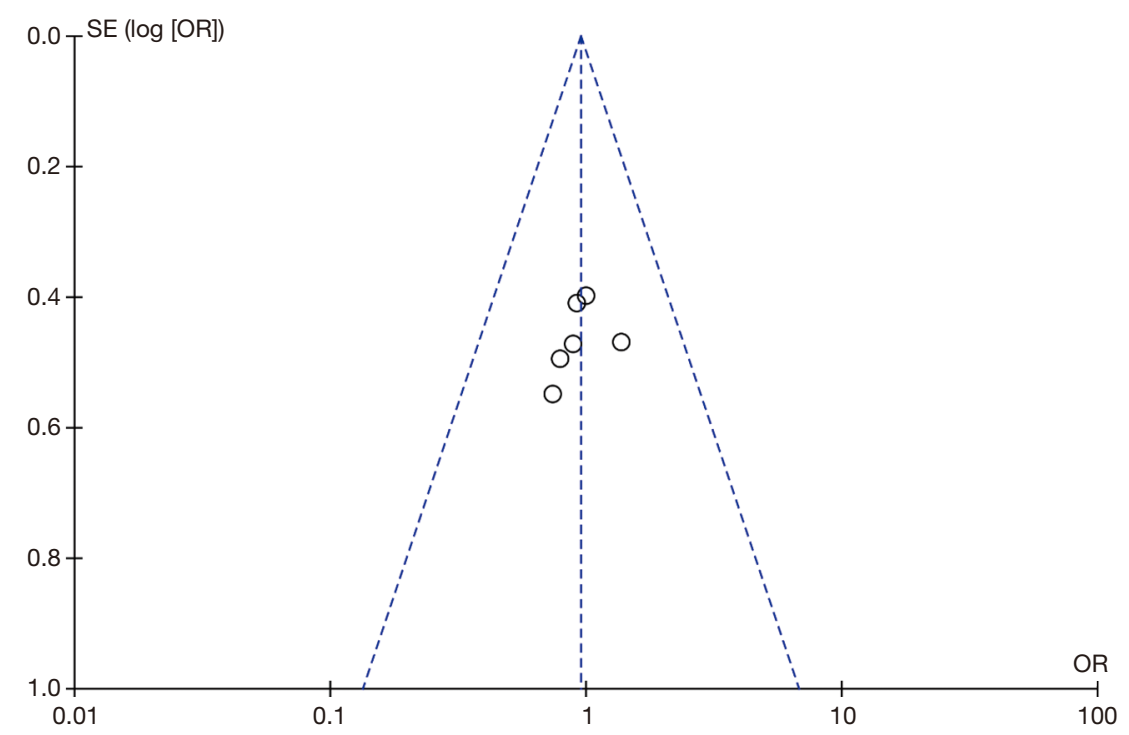

Figure 14 A funnel chart showing the recurrence rate between patients administered anti-thymocyte globulin (ATG) combined with eltrombopag and patients given ATG alone.

human thymocytes into animals such as rabbits and horses. The animals produce specific antibodies against the human thymocytes (17). These resultant anti-thymocyte antibodies can then be used for immunosuppressive therapy in patients with SAA. Lengline et al. (18) suggested that the effect of combined immunosuppressive therapy was more extensive than that of single-drug therapy, and that synergy could be easily achieved.

A total of 16 publications were selected from Chinese and English medical databases. The quality classification results of the meta-analysis showed that 8 references scored 6-9 or above, 5 references scored 2-5, and 3 references scored 2 points and below. Among the $16 \mathrm{RCTs}, 3$ had a correct random allocation method, with only 2 adopting allocation concealment.

In this study, patients treated with ATG combined with eltrombopag were compared to patients administered ATG alone. The total effective rate, survival rate, mortality, side effects, and recurrence rate were examined. Meta-analysis results showed that patients administered ATG combined with eltrombopag had higher effective rate, greater survival rate, lower mortality, and lower incidence of side effects. While the recurrence rate of patients in the ATG combined with eltrombopag group was slightly lower than that of patients in the ATG alone group, this was not statistically significant and may be caused by publication bias.

There are some limitations to this study. Several of the references included were of low quality and had high 
heterogeneity, and the sample size was small. Hence, further verification using larger numbers of clinical RCTs is warranted.

\section{Conclusions}

In this meta-analysis, 16 studies were included using the Boolean logic search method, with combined ATG and eltrombopag therapy as the experimental group and ATG therapy alone as the control group. The investigation confirmed that the application of ATG combined with eltrombopag in the treatment of SAA was safer and more effective than ATG alone. The results of this study provide a reliable theoretical basis for the clinical treatment of SAA.

\section{Acknowledgments}

Funding: None.

\section{Footnote}

Reporting Checklist: The authors have completed the PRISMA reporting checklist. Available at http://dx.doi. org/10.21037/apm-21-1049

Conflicts of Interest: All authors have completed the ICMJE uniform disclosure form (available at http://dx.doi. org/10.21037/apm-21-1049). The authors have no conflicts of interest to declare.

Ethical Statement: The authors are accountable for all aspects of the work in ensuring that questions related to the accuracy or integrity of any part of the work are appropriately investigated and resolved.

Open Access Statement: This is an Open Access article distributed in accordance with the Creative Commons Attribution-NonCommercial-NoDerivs 4.0 International License (CC BY-NC-ND 4.0), which permits the noncommercial replication and distribution of the article with the strict proviso that no changes or edits are made and the original work is properly cited (including links to both the formal publication through the relevant DOI and the license). See: https://creativecommons.org/licenses/by-nc-nd/4.0/.

\section{References}

1. Bacigalupo A. Antithymocyte globulin and cyclosporin: standard of care also for older patients with aplastic anemia. Haematologica 2019;104:215-6.

2. Du S, He W, Yang Y, et al. Unrelated cord blood transplantation for severe aplastic anemia using intensified immunoablative conditioning regimen leading to high engraftment and survival. Bone Marrow Transplant 2020;55:1677-80.

3. Kekre N, Zhang Y, Zhang MJ, et al. Effect of antithymocyte globulin source on outcomes of bone marrow transplantation for severe aplastic anemia. Haematologica 2017;102:1291-8.

4. Tichelli A, de Latour RP, Passweg J, et al. SAA Working Party of the EBMT. Long-term outcome of a randomized controlled study in patients with newly diagnosed severe aplastic anemia treated with antithymocyte globulin and cyclosporine, with or without granulocyte colonystimulating factor: a Severe Aplastic Anemia Working Party Trial from the European Group of Blood and Marrow Transplantation. Haematologica 2020;105:1223-31.

5. Rogers ZR, Nakano TA, Olson TS, et al. Immunosuppressive therapy for pediatric aplastic anemia: a North American Pediatric Aplastic Anemia Consortium study. Haematologica 2019;104:1974-83.

6. Hayakawa J, Kanda J, Akahoshi Y, et al. Meta-analysis of treatment with rabbit and horse antithymocyte globulin for aplastic anemia. Int J Hematol 2017;105:578-86.

7. Yang N, Chen J, Zhang H, et al. Horse versus rabbit antithymocyte globulin in immunosuppressive therapy of treatment-naïve aplastic anemia: a systematic review and meta-analysis. Ann Hematol 2017;96:2031-43.

8. Kekre N, Zhang Y, Zhang MJ, et al. Effect of antithymocyte globulin source on outcomes of bone marrow transplantation for severe aplastic anemia. Haematologica 2017;102:1291-8.

9. Lengline E, Drenou B, Peterlin P, et al. Nationwide survey on the use of eltrombopag in patients with severe aplastic anemia: a report on behalf of the French Reference Center for Aplastic Anemia. Haematologica 2018;103:212-20.

10. Sasaki N, Shimura K, Yoshida M, et al. Kyoto Clinical Hematology Study Group (KOTOSG) investigators. Immunosuppressive therapy with rabbit antithymocyte globulin therapy for acquired aplastic anemia: a multiinstitutional retrospective study in Japanese adult patients. Int J Hematol 2019;109:278-85.

11. Clé DV, Atta EH, Dias DSP, et al. Rabbit antithymocyte globulin dose does not affect response or survival as firstline therapy for acquired aplastic anemia: a multicenter retrospective study. Ann Hematol 2018;97:2039-46. 
12. Admiraal R, de Witte MA, Huitema A, et al. Pharmacological Considerations in Antithymocyte Globulin Exposure Calculation. Biol Blood Marrow Transplant 2019;25:e213-e215.

13. Lin SF, Liu S, Xue HM, et al. Comparison of two dosages of rabbit antithymocyte globulin (r-ATG) in treating children with severe aplastic anemia. Pharmazie 2018;73:264-8.

14. Ochi T, Onishi Y, Nasu K, et al. Umbilical Cord Blood Transplantation Using Reduced-Intensity Conditioning without Antithymocyte Globulin in Adult Patients with Severe Aplastic Anemia. Biol Blood Marrow Transplant 2019;25:e55-e59.

15. Fu R, Chen T, Song J, et al. De-escalation empirical antibiotic therapy improved survival for patients with severe aplastic anemia treated with antithymocyte globulin. Medicine (Baltimore) 2017;96:e5905.

Cite this article as: Zhang J, Wu Y, Liu J, Han S, Chen L, Wang H, Peng Y. A systematic review and meta-analysis of the safety and efficacy of anti-thymocyte globulin combined with eltrombopag in the treatment of severe aplastic anemia. Ann Palliat Med 2021;10(5):5549-5560. doi: 10.21037/apm-21-1049
16. Vallejo C, Montesinos P, Polo M, et al. Bone Marrow Failure Spanish Study Group (Pethema-GETH). Rabbit antithymocyte globulin versus horse antithymocyte globulin for treatment of acquired aplastic anemia: a retrospective analysis. Ann Hematol 2015;94:947-54.

17. Chen X, Wei J, Huang Y, et al. Effect of Antithymocyte Globulin Source on Outcomes of HLA-Matched Sibling Allogeneic Hematopoietic Stem Cell Transplantation for Patients with Severe Aplastic Anemia. Biol Blood Marrow Transplant 2018;24:86-90.

18. Lengline E, Drenou B, Peterlin P, et al. Nationwide survey on the use of eltrombopag in patients with severe aplastic anemia: a report on behalf of the French Reference Center for Aplastic Anemia. Haematologica 2018;103:212-20.

(English Language Editor: J. Teoh) 